\title{
The Role of a Medical Intermediate Care Unit in the Management of Budd-Chiari Syndrome: Case Series
}

\author{
Marcia Cravo $^{\mathrm{a}, \mathrm{f}}$, Daniel G. Oliveira ${ }^{\mathrm{a}}$, Arlindo Guimas ${ }^{\mathrm{b}}$, Pedro Vita ${ }^{\mathrm{b}}$, Ana Luisa Rego ${ }^{\mathrm{b}}$, \\ Rute Alves $^{\mathrm{b}}$, Diana Valadares ${ }^{\mathrm{b}}$, Graziela Carvalheiras ${ }^{\mathrm{b}}$, Miguel Ricardo ${ }^{\mathrm{b}}$, \\ Alexandre Pinto ${ }^{\mathrm{b}, \mathrm{c}}$, Helena Pessegueiro ${ }^{\mathrm{d}}$, Filipe Nery , c, e
}

\begin{abstract}
Budd-Chiari syndrome (BCS) has a wide spectrum of presentations, from an asymptomatic status to acute liver failure (ALF). The therapeutic approach depends on disease severity and related etiology with patients with severe forms of presentation classically managed in intensive care units (ICUs). Here, we report a series of five BCS patients managed in a medical intermediate care unit (IntCU), with three of them presenting with acute liver injury. Progression to ALF was seen in three patients, two of whom died, with one being successfully submitted to liver transplantation. IntCUs allow a 24-h patient surveillance and a prompt management of BCS, with less economic impact when compared to ICUs. Mortality was related to the presence of associated comorbidities that limited therapeutic approach.
\end{abstract}

Keywords: Acute liver failure; Budd-Chiari syndrome; Hypercoagulable state

\section{Introduction}

Budd-Chiari syndrome (BCS) is a rare condition that refers to any obstruction of the hepatic venous outflow from the hepatic venules to the junction of the inferior vena cava (IVC) with the right atrium $[1,2]$. Several causes contribute to BCS, usually being recognized as an underlying prothrombotic condition.

Manuscript submitted January 24, 2021, accepted February 1, 2021

Published online March 5, 2021

aServico de Medicina Interna, Centro Hospitalar Universitario do Porto, Porto, Portugal

${ }^{b}$ Unidade Intermedia Medica, Servico de Cuidados Intensivos, Centro Hospitalar Universitario do Porto, Porto, Portugal

'Instituto de Ciencias Biomedicas Abel Salazar-Universidade do Porto, Porto, Portugal

dUnidade de Transplante Hepato-Pancreatica, Centro Hospitalar Universitario do Porto, Porto, Portugal

eEpiUnit, Instituto de Saude Publica da Universidade do Porto, Porto, Portugal ${ }^{f}$ Corresponding Author: Marcia Cravo, Servico de Medicina Interna, Centro Hospitalar Universitario do Porto, Largo Prof. Abel Salazar S/N, Porto 4099001, Portugal. Email: marciacravosilva@gmail.com

doi: https://doi.org/10.14740/jmc3659
Etiological work-up must be promptly conducted, as concomitant treatment towards the underlying disease is mandatory [1-4]. Therapeutic strategies depend on the disease severity and underlying etiology, with anticoagulation (Aco) being the mainstay. Validated survival scores are of little utility [5].

$\mathrm{BCS}$ has a wide spectrum of presentations $[2,6]$. Patients progressing to acute liver failure (ALF) are exceptional but with high in-hospital mortality rates. The scarcity of published data contributes to discrepant approaches and eventually different outcomes, depending on local experience [6]. No mention of BCS management in medical intermediate care units (IntCUs) has been made so far.

The aim of this study was to retrospectively report on clinical presentation, stratification, therapeutic strategies, prognostic scores, and outcome of a series of five cases of symptomatic BCS admitted to an IntCU of a tertiary hospital over a 9-year period.

\section{Case Reports}

\section{Case 1}

An 18-year-old female with history of autoimmune hepatitis and triple positive antiphospholipid syndrome (APS) under Aco treatment with acenocoumarol, presented at the emergency room with acute abdominal pain. She was afebrile, hemodynamically stable. No clinical ascites or hepatic encephalopathy was documented. At admission, renal and liver biochemical profiles were unremarkable. She presented an international normalized ratio (INR) of 4.8 (under vitamin $\mathrm{K}$ antagonist) with related Child-Pugh (CP) classification of 7 points (B) (considering the INR range under acenocoumarol). Abdominal Doppler ultrasonography (Doppler-US) revealed a $6 \mathrm{~cm}$ thrombus extending from the IVC to near the entrance of the right atrium, confirmed by computed tomography (CT) scan, with extension to all the three supra-hepatic veins. Effective collateral venous circulation was present through the lumbar and azygos veins. Diagnosis of sub-acute on chronic BCS related to triple positive APS was established. As the patient was under vitamin $\mathrm{K}$ antagonist, treatment with non-fractioned heparin (NFH) was started together with low-dose acetylsalicylic acid. Treatment directed towards APS with plasmapheresis and rituximab was done. The patient improved clinically 
and was discharged under acenocoumarol with INR target values for 2.5 - 3.5 and low-dose acetylsalicylic acid.

\section{Case 2}

A 29-year-old female with previous history of APS with peripheral venous thrombosis presented to the emergency room with acute abdominal pain and abdominal distension, after suspending Aco treatment with acenocoumarol for 4 days. She presented with grade 2 ascites and no evidence of hepatic encephalopathy. Laboratory data on admission revealed an altered liver profile with alanine aminotransferase (ALT) of 82 $\mathrm{U} / \mathrm{L}$ (upper normal limit $(\mathrm{UNL})=36$ ), aspartate aminotransferase $(\mathrm{AST})$ of $138 \mathrm{U} / \mathrm{L}(\mathrm{UNL}=30)$, alkaline phosphatase $($ ALP) of $130 \mathrm{U} / \mathrm{L}(\mathrm{UNL}=104)$, gamma glutamyl transferase $(\mathrm{GGT})$ of $56 \mathrm{U} / \mathrm{L}(\mathrm{UNL}=39)$ and total bilirubin $(\mathrm{TB})$ of 4.02 $\mathrm{mg} / \mathrm{dL}$ with direct bilirubin (DB) of $2.43 \mathrm{mg} / \mathrm{dL}$; renal function was normal. An initial INR of 2.72 (4 days after discontinuing Aco) was found, resulting in a model of end-stage liver disease (MELD) score of 23, model of end-stage liver disease-sodium (MELD-Na) score of 26 and a CP C score of 10 points. Abdominal Doppler-US presented thrombus in all three supra-hepatic veins and ascites, both confirmed by CT scan. There were no documented collaterals. Diagnosis of acute BCS related to APS was made and treatment with NFH was started. By day 3 of hospitalization she had evolved unfavorably with an INR of 4.45, AST of $549 \mathrm{U} / \mathrm{L}$, ALT of $110 \mathrm{U} / \mathrm{L}$, TB of $8.80 \mathrm{mg} / \mathrm{dL}$ and ALP of $110 \mathrm{U} / \mathrm{L}$. Due to this progression to acute liver injury (ALI) reflected by worsening of MELD/MELD-Na of 31 and CP C score of 12 points, angioplasty was performed. Pain was controlled initially with opioids and ascites with diuretic treatment. After vascular procedure, laboratory changes improved and pain was controlled. The patient was discharged under acenocoumarol treatment for INR target of 2.5 - 3.5.

\section{Case 3}

A healthy 29-year-old male presented at the emergency room with acute abdominal pain, fever and nausea. He was hemodynamically stable, with ascites and no signs of hepatic encephalopathy. Laboratory study revealed lymphocytes of $10,610 / \mu \mathrm{L}$, hemoglobin of $6.6 \mathrm{~g} / \mathrm{dL}$, platelets of $8,000 / \mu \mathrm{L}, \mathrm{sCr}$ of $1.19 \mathrm{mg} /$ $\mathrm{dL}$ and sodium of $134 \mathrm{mmol} / \mathrm{L}$. Liver tests demonstrated ALT of $3,797 \mathrm{U} / \mathrm{L}$, AST of $3,967 \mathrm{U} / \mathrm{L}$, TB of $2.49 \mathrm{mg} / \mathrm{dL}$ (DB of $1.8 \mathrm{mg} / \mathrm{dL}$ ), albuminemia of $2.6 \mathrm{mg} / \mathrm{dL}$ and INR of 4.1 . He presented, at admission, with a CP C score of 11 points, MELD of 27 points and MELD-Na of 28. Abdominal Doppler-US revealed ascites and thrombus in all three supra-hepatic veins confirmed by CT scan. Spontaneous bacterial peritonitis was excluded. ALI related to BCS was established. Due to severe thrombocytopenia, Aco was not started. Etiologic work-up led to an inaugural diagnosis of acute myeloid leukemia (AML). The patient rapidly developed ALF with hepatic encephalopathy and acute renal failure with peak $\mathrm{sCr}$ of $1.8 \mathrm{mg} / \mathrm{dL}$. Acute leukemia contraindicated liver transplant (LT) and liver dysfunction pre-empted any treatment for leukemia. Palliative care was started to control pain and the patient died 2 days later.

\section{Case 4}

A 51-year-old female with a history of primary myelofibrosis and dilated cardiomyopathy presented at the emergency room with acute abdominal pain. She was afebrile and hemodynamically stable. No signs of hepatic encephalopathy were present. Laboratory study at first evaluation revealed leucocytes of $25,570 / \mu \mathrm{L}$, hemoglobin of $13.9 \mathrm{~g} / \mathrm{dL}$, platelets of $324,000 / \mu \mathrm{L}$, $\mathrm{sCr}$ of $2.45 \mathrm{mg} / \mathrm{dL}, \mathrm{AST}$ of $126 \mathrm{U} / \mathrm{L}, \mathrm{ALT}$ of $131 \mathrm{U} / \mathrm{L}, \mathrm{ALP}$ of $413 \mathrm{U} / \mathrm{L}, \mathrm{GGT}$ of $220 \mathrm{U} / \mathrm{L}, \mathrm{TB}$ of $11.5 \mathrm{mg} / \mathrm{dL}$ (DB of $9.67 \mathrm{mg} /$ $\mathrm{dL}$ ), albumin of $2.7 \mathrm{mg} / \mathrm{dL}$, INR of 1.95 and configuring $\mathrm{CP}$ $\mathrm{C}$ score of 11 points and MELD and MELD-Na score of 31 and 33 points, respectively. Abdominal Doppler-US showed thrombus in all the three supra-hepatic veins and ascites, confirmed by CT scan. Spontaneous bacterial peritonitis was excluded. The patient was diagnosed with an ALI related to BCS secondary to primary myelofibrosis and she was started on Aco therapy with NFH along with an antiplatelet agent and cytoreductive therapy with hydroxyurea. At day 7, the patient developed ALF with grade 3 West-Haven encephalopathy. CP $\mathrm{C}$ score at that time was 13 points. Transjugular intra-hepatic portosystemic shunting (TIPS) was contraindicated due to cardiac failure, the presence of relevant encephalopathy and high MELD score (MELD 34/MELD-Na 35, under NFH). LT was considered as a high-risk surgery and contraindicated after multidisciplinary discussion. The patient died due to liver failure 2 days after beginning palliative care.

\section{Case 5}

A 47-year-old male with a history of repeated peripheral venous thrombosis with no established etiology, without chronic Aco therapy, presented to the emergency room with acute abdominal pain and grade 1 West-Haven hepatic encephalopathy. Laboratory studies revealed lymphocytes of $8,110 / \mu \mathrm{L}$, hemoglobin of $15.9 \mathrm{~g} / \mathrm{dL}$, platelets of $183,000 /$ $\mu \mathrm{L}$, sCr of $0.78 \mathrm{mg} / \mathrm{dL}$, sodium of $130 \mathrm{mmol} / \mathrm{L}$, ALT of 194 $\mathrm{U} / \mathrm{L}, \mathrm{AST}$ of $233 \mathrm{U} / \mathrm{L}$, TB of $7.20 \mathrm{mg} / \mathrm{dL}$ (DB of $4.3 \mathrm{mg} /$ $\mathrm{dL}$ ), albumin of $3 \mathrm{mg} / \mathrm{dL}$ and INR of 2.6 resulting in a $\mathrm{CP}$ $\mathrm{C}$ score of 11 points, MELD-Na of 28 points and MELD 25 points. CT scan revealed an acute thrombus of the supra-hepatic veins and chronic thrombosis of the IVC, ascites and homogeneous splenomegaly. Spontaneous bacterial peritonitis was excluded. NFH and antiplatelet therapy were started. A myeloproliferative disorder was ruled out (myeloproliferative leukemia virus (MPL), calreticulin (CALR) and exon 12 of Janus kinase (JAK) 2 mutations were negative), as were APS, Behcet's disease, paroxysmal nocturnal hemoglobinuria. Factor $\mathrm{V}$ Leiden and prothrombin gene mutations were negative. Homocysteine levels were normal. The patient was successfully submitted to LT (angioplasty/stenting or TIPS was not considered as an option due to technical concerns raised by the interventional radiology team). 


\section{Discussion}

Although it is rare, BCS must be considered in acute or chronic liver diseases, particularly when other causes have been excluded and in the acute onset of ascites and abdominal pain [3]. In about $90 \%$ cases, at least one underlying risk factor is identified, reinforcing the need of an intensive etiological work-up. However, some predisposing disorders only manifest after the initial clinical presentation [1]. We found BCS related to two APS, one newly diagnosed AML and one primary myelofibrosis, with only one patient not having had an identified precipitating factor (Table 1).

Clinical manifestations are wide depending on the extension of the hepatic-vein occlusion and on the efficiency of venous collateral circulation $[2,3]$. In our case series, acute abdominal pain was present in all patients, three of them with ascites. One patient had hepatic encephalopathy at admission while two others developed that during hospitalization. Evolution to ALF was more common than previously reported (> $50 \%$ of patients), a perspective that may be biased as more severe patients are admitted in IntCU than in general wards. The presence of effective venous collateral circulation, as seen in one patient, may relate to a less severe form of presentation and better outcome.

Investigation aims to establish BCS diagnosis, assess liver injury and disease severity and underlie etiology. CP score and peak of ALT and renal function are all important to determinate prognosis $[6,7]$. Routine biochemical analyses have little diagnostic value in BCS $[2,8]$. In the presented cases, only the patient with sub-acute on chronic form of BCS presented with normal liver profile. In others, cytolysis (ALT between 3.6 and 105 times the upper normal limit) and coagulopathy were the usual findings (Table 1). In our series, the majority had high $\mathrm{CP}$ admission scores, except for case 1 . Both cases 3 and 4, the first with the highest ALT value and the second with the worse renal function, died in $48 \mathrm{~h}$ after progression to ALF. The dismal outcome of these two patients was related to underlying diseases, limiting therapeutic approaches. In cases 2 and 5, despite increased ALT and CP scores, prompt treatment with Aco, angioplasty and LT (respectively), was liable for a better prognosis. Of notice, in all patients the diagnosis was done with Doppler-US, while CT scan confirmed the diagnosis and checked for effective collateral circulation through the azygos system.

Therapeutic strategies are defined by disease severity and depend on local expertise. All patients diagnosed with BCS should receive Aco in the absence of contraindications, and specific treatment to the underlying prothrombotic condition $[2,9]$. Portal hypertension complications should be treated [9]. Symptomatic patients that do not improve with medical treatment or develop severe forms of BCS should be considered for angioplasty/stenting or even TIPS [7, 9]. LT should be considered if liver dysfunction progresses $[3,5,9]$. General and specific treatments and respective outcomes are expressed in Table 1. All patients were started on Aco with NFH, except for case 3 due to severe thrombocytopenia. Case 1 (sub-acute form) was treated with medical therapy towards APS with plasmapheresis and rituximab, as the effectiveness of the collateral venous cir-

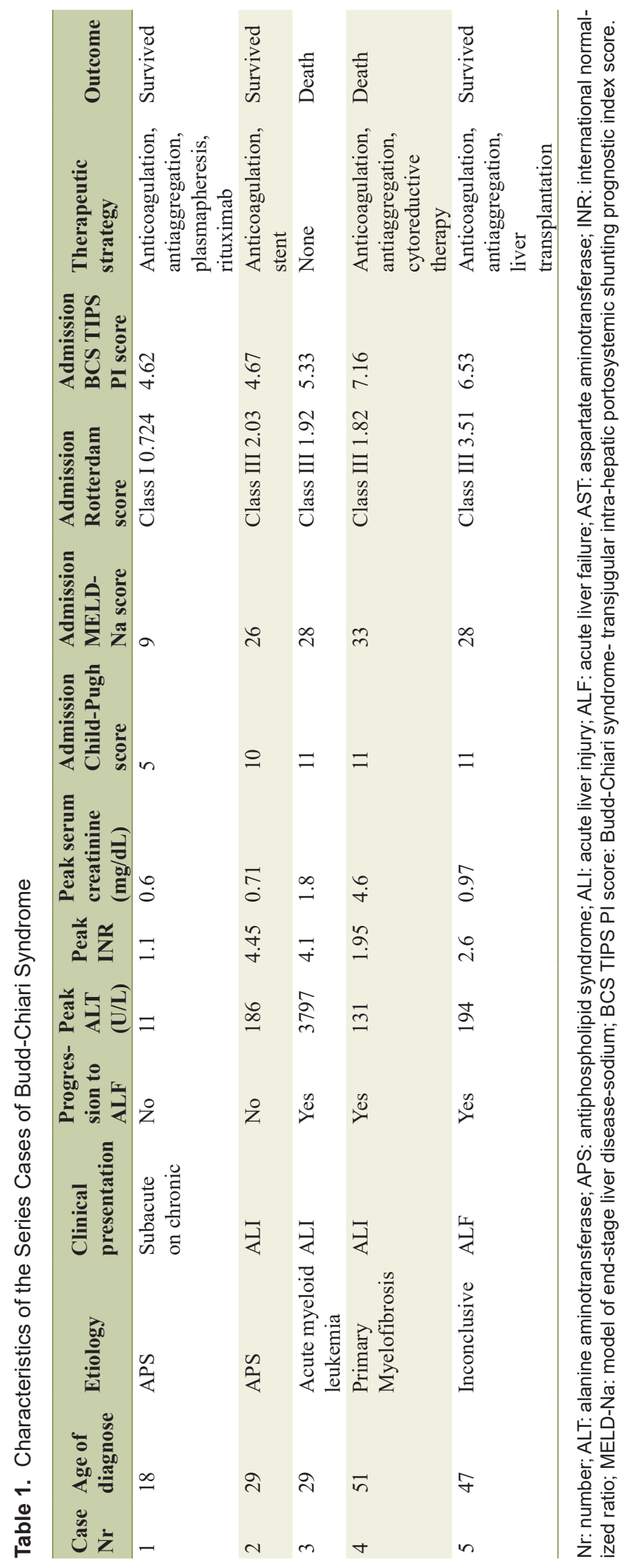


culation possibly prevented liver failure. The other case of BCS with underlying APS (case 2) rapidly evolving to ALI, in the absence of an effective collateral circulation, required a derivation technique. In the case of ALI with underlying myeloproliferative syndrome (case 4), it required other approaches such as cytoreduction. In case 3 , no treatment towards the newly diagnosed acute leukemia was possible due to ALF, and LT was ruled out due to active malignancy. Finally, the patient received transplantation due to ALF, after underwent Aco without clinical improvement and reperfusion techniques were not considered feasible by experienced radiologists.

Our experience demonstrates that it is the contraindication to recommended treatments that seems to culminate in mortality, instead of the severity of the disease expressed by BCS scores (Table 1).

All these patients were managed in a medical, level 2, IntCU from the very beginning, allowing a 24-h nurse and medical surveillance. Our IntCU has the particularity of being physically side-by-side function of an ICU and of lying in a tertiary hospital with a dedicated liver transplantation team, granting the proper and safe handling of these patients. Dedicated IntCUs are adequate places for the clinical management of BCS patients and represent a more cost-efficient alternative to ICUs, with two-third lower costs per day when comparing both infrastructures. Unfortunately, it was not possible to compare our small series to a historical cohort of BCS patients admitted in ICU, regarding outcomes, hospitalization days, nosocomial complications and final total costs.

\section{Conclusion}

While being rare, severe BCS patients are usually managed in ICUs and their management depends on the experience and facilities of the center. Medical IntCUs are now proliferating and occupying their central role and position in hospitals, as they can manage severe ill patients that do not require invasive respiratory support, with a 24-h surveillance. Acute BCS patients could be admitted in IntCU as a proper place to etiological investigation and prompt treatment and management of these patients, with reduced costs when comparing to an ICU admission, which also play an important role when deciding proper allocation. We also found with this case series that, even though patients died from complications of liver failure, contraindication to adequate treatment was the most relevant variable to the outcome.

IntCUs may be efficient cost-effective places in the approach of this disorder.

\section{Acknowledgments}

None to declare.

\section{Financial Disclosure}

This research did not receive any specific grant from funding agencies in the public, commercial, or not-for-profit sectors.

\section{Conflict of Interest}

All the authors declare that no conflict of interest exists.

\section{Informed Consent}

Informed consent was obtained from each one of the three patients that are still alive, as well as one of the patients that, being dead, we achieved to obtain it with the next of kin. It was impossible to obtain a written informed consent from one of the patients, that also died, or his family. Ethic committee authorized it publication. All the signed informed consents are in the possession of the first author.

\section{Author Contributions}

Cravo $\mathrm{M}$ and Nery F, conceived of the presented idea and developed it. All authors contributed to the acquisition of data. Cravo $\mathrm{M}$ and Nery $\mathrm{F}$ wrote the manuscript with input from all authors. All authors discussed the results and contributed to the final manuscript.

\section{Data Availability}

The authors declare that data supporting the findings of this study are available within the article.

\section{References}

1. Aydinli M, Bayraktar Y. Budd-Chiari syndrome: etiology, pathogenesis and diagnosis. World J Gastroenterol. 2007;13(19):2693-2696.

2. Menon KV, Shah V, Kamath PS. The Budd-Chiari syndrome. N Engl J Med. 2004;350(6):578-585.

3. Plessier A, Valla DC. Budd-Chiari syndrome. Semin Liver Dis. 2008;28(3):259-269.

4. Darwish Murad S, Plessier A, Hernandez-Guerra M, Fabris F, Eapen CE, Bahr MJ, Trebicka J, et al. Etiology, management, and outcome of the Budd-Chiari syndrome. Ann Intern Med. 2009;151(3):167-175.

5. Plessier A, Rautou PE, Valla DC. Management of hepatic vascular diseases. J Hepatol. 2012;56(Suppl 1):S2538.

6. Parekh J, Matei VM, Canas-Coto A, Friedman D, Lee WM, Acute Liver Failure Study Group. Budd-chiari syndrome causing acute liver failure: A multicenter case series. Liver Transpl. 2017;23(2):135-142.

7. Janssen HL, Garcia-Pagan JC, Elias E, Mentha G, Hadengue A, Valla DC, European Group for the Study of Vascular Disorders of the Liver. Budd-Chiari syndrome: a review by an expert panel. J Hepatol. 2003;38(3):364- 
371.

8. Mitchell MC, Boitnott JK, Kaufman S, Cameron JL, Maddrey WC. Budd-Chiari syndrome: etiology, diagnosis and management. Medicine (Baltimore). 1982;61(4):199-
218.

9. European Association for the Study of the Liver. EASL Clinical Practice Guidelines: Vascular diseases of the liver. J Hepatol. 2016;64(1):179-202. 